\title{
Complex Frontal Upper Teeth Injury with Children - A Case Study of Preconditions for Efficient Procedure of Such an Injury Treatment
}

\author{
Dusan Surdilovic* \\ Department for Paediatric Dentistry and Orthodontics, Gulf Medical University, UAE
}

Submission: April 28, 2017; Published: March 19, 2018

*Corresponding author: Dusan Surdilovic, Department for Paediatric Dentistry and Orthodontics, HOD, Gulf Medical University, Ajman, Dubai, UAE, Email: surdilovic74@gmail.com

Abstract

Traumatic extraction of permanent teeth (Avulsion Completa) is a serious tooth injury, having an uncertain outcome and requiring a quick and proper reaction of the dentist. In this study, a full therapy plan for an 11 year old girl was shown, who came to the clinic with avulsed tooth 11, third class fracture of tooth 12 and second class fracture of tooth 21 . After clinical examination and upper frontal region X-ray, replantation of tooth 11 was applied, as well as wire composite splint placement. Replantation and injured teeth endodontic treatment being successfully performed, a prosthetic sanation was done next on the patient. In the study, a clinical picture of the patient one year after the injury was also shown.

Keywords: Avulsion; Replantation; Splint

\section{Introduction}

Traumatic tooth extraction (Avulsion Completa, Extrusion Completa, Exarticulation) is a rare injury of both milk and permanent teeth, but more frequently seen in the former. The most common traumatic extraction in milk teeth occurs as a result of falls, whereas in permanent teeth, the main etiological factor is direct (frontal) hitting. The most often disrupted teeth are central upper incisors. The frequency of this injury compared to others is very small, rating about $0.9 \%$ of total teeth injuries.

Traumatic tooth extraction can clinically be seen as a tooth missing from the teeth line. Diagnostically same or similar clinical picture can be seen in tooth extrusion and tooth root fracture with tooth crown loss as well, therefore an X-ray being obligatory for the final diagnosis.

Tooth replantation success depends on numerous factors: Time between traumatic extraction and replantation, the way of storing the tooth from avulsion until replantation, the level of the avulsed tooth root fracture, alveolar bone condition and prior condition of the tooth crown, pulp and periodontium, possible orthodontic irregularities and, as a very important factor, the procedure during replantation.

\section{Case Report}

On July 10th 2015, a patient M.K. (born in 2004) came to the dentistry clinic with her father due to a multiple tooth injury after a bicycle fall in which she hit directly the handlebar with her face. The girl's father brought the avulsed tooth 11 in a cotton tissue. Almost 2 hours passed between the time when the injury occurred and her admission at the clinic. After taking the patient's history, clinical examination and the X-ray (Figure 1), the following diagnosis was made: 11 avulsion completa dentis traumatica; 12 fracture III class dentis traumatica; fracture II class dentis traumatic; 22 contusio dentis. Tooth 11 also had II class fracture Figure 1.

Immediately after admission, tooth 11 was put into physiological solution. Decolorisation of the upper frontal region was done with $2 \mathrm{ml}$ of Lidokain anesthetic. Afterwards, we started wire composite splint placement on teeth 15, 14, 21, 22, 24 (Figure 2).

After the splint placement, alveoli were rinsed with coagulum physiological solution followed by a manual replantation of the traumatically avulsed tooth 11 . Then, tooth 11 was bonded with the already placed wire composite splint $(15,14,11,21,22$ and 24) (Figure 3). The emphasis of the whole procedure is on TIME, as only $2 \mathrm{~h} 7 \mathrm{~min}$ passed from the moment of injury until replantation. After splint placement, a control X-ray was done (Figure 4). During this first visit, vital extirpation of tooth 12 pulps was done and the tooth endodontic treatment was applied with calcium hydroxide paste - Calcipupe Septodont, Cedex, France. A composite bandage was put on tooth 21 . Gingival tooth 11 sulcus 


\section{Advances in Dentistry \& Oral Health}

was rinsed with Orvagil solution (metronidazol). The patient was given an antibiotic therapy as well (Figure $3 \& 4$ ).

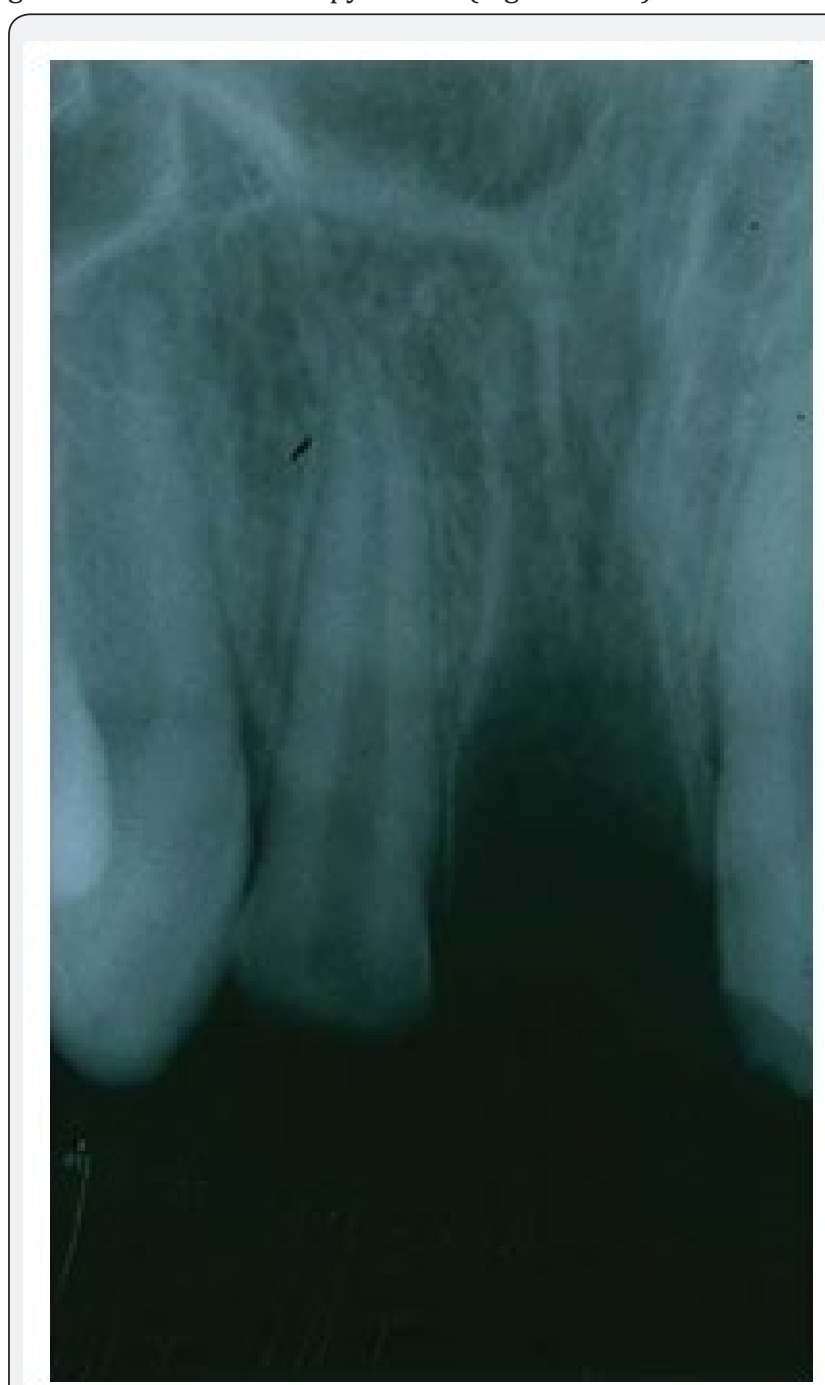

Figure 1

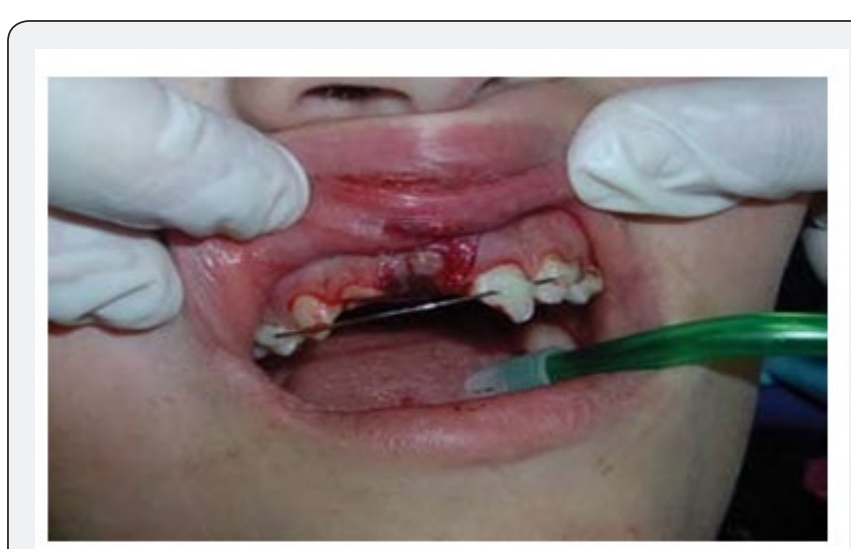

Figure 2.
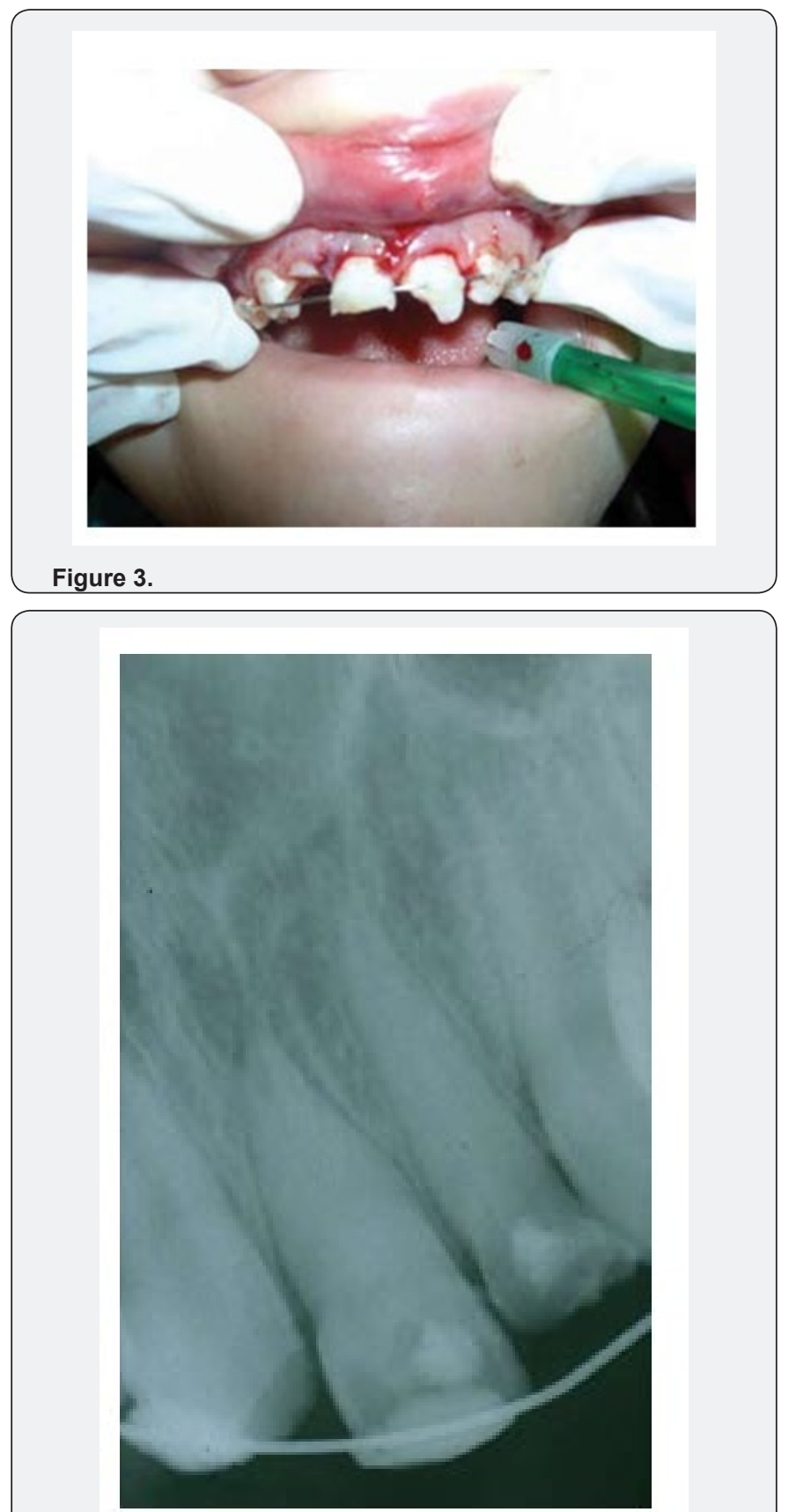

Figure 4.

24 hours after the injury, tooth 11 pulp extirpation was done, performing endodontic treatment of tooth root canal with calcium hydroxide paste. Tooth 21 vitality was regained on the 4 th day after the injury. Further checkups were scheduled every seven days, during which revisions of fillings in teeth 11 and 12 were done with the previously mentioned paste.

Prior to the splint removal, on the 21st day after the injury, the final filling of tooth 11 was done (Endomethasone Septodent, Cedex, France). After both final fillings, a control X-ray image was 


\section{Advances in Dentistry \& Oral Health}

made (Figure 5). On the 28th day after the placement, the wire composite splint was removed (Figure 6).
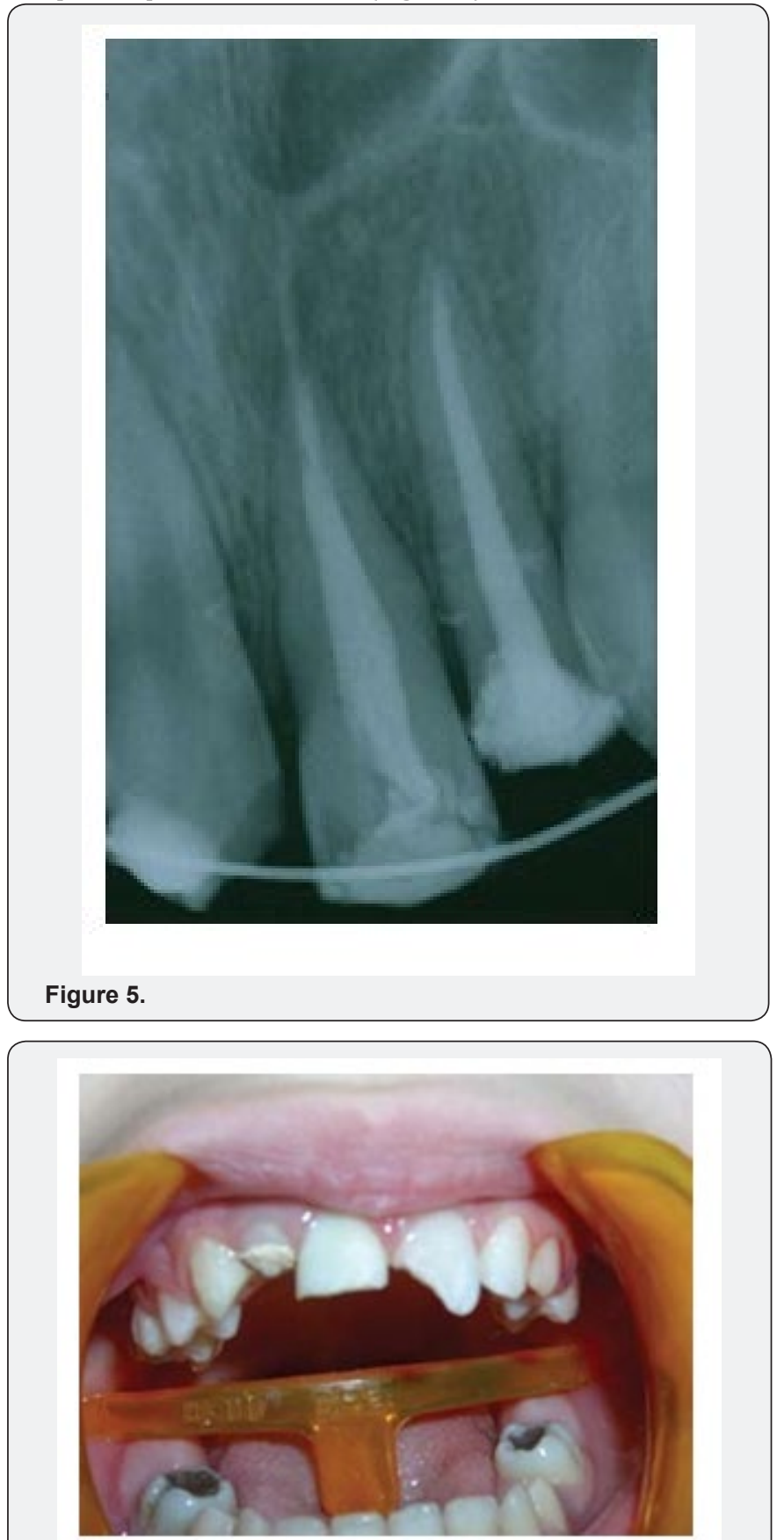

Figure 6.

After splint removal, the bonded teeth in the splint were treated with Fluorogal forte gel according to the protocol for patients with high risk of cavity. The final composite restoration of teeth 11 and 21 was done using restorative materials Te-Econom Ivoclar - vivadent. Three months after the injury, one more control X-ray image was made (Figure 7), showing regular clinical findings with anamnesis data not showing any subjective or objective discomfort in the patient.
Three months later, a prosthetic dental ceramic crown portion for tooth 12 was placed. A year later, the clinical findings were neat, with no pathological changes. Following of the patient's medical state is obligatory and the checks up examinations are scheduled every three months (Figure 8 \& 9).
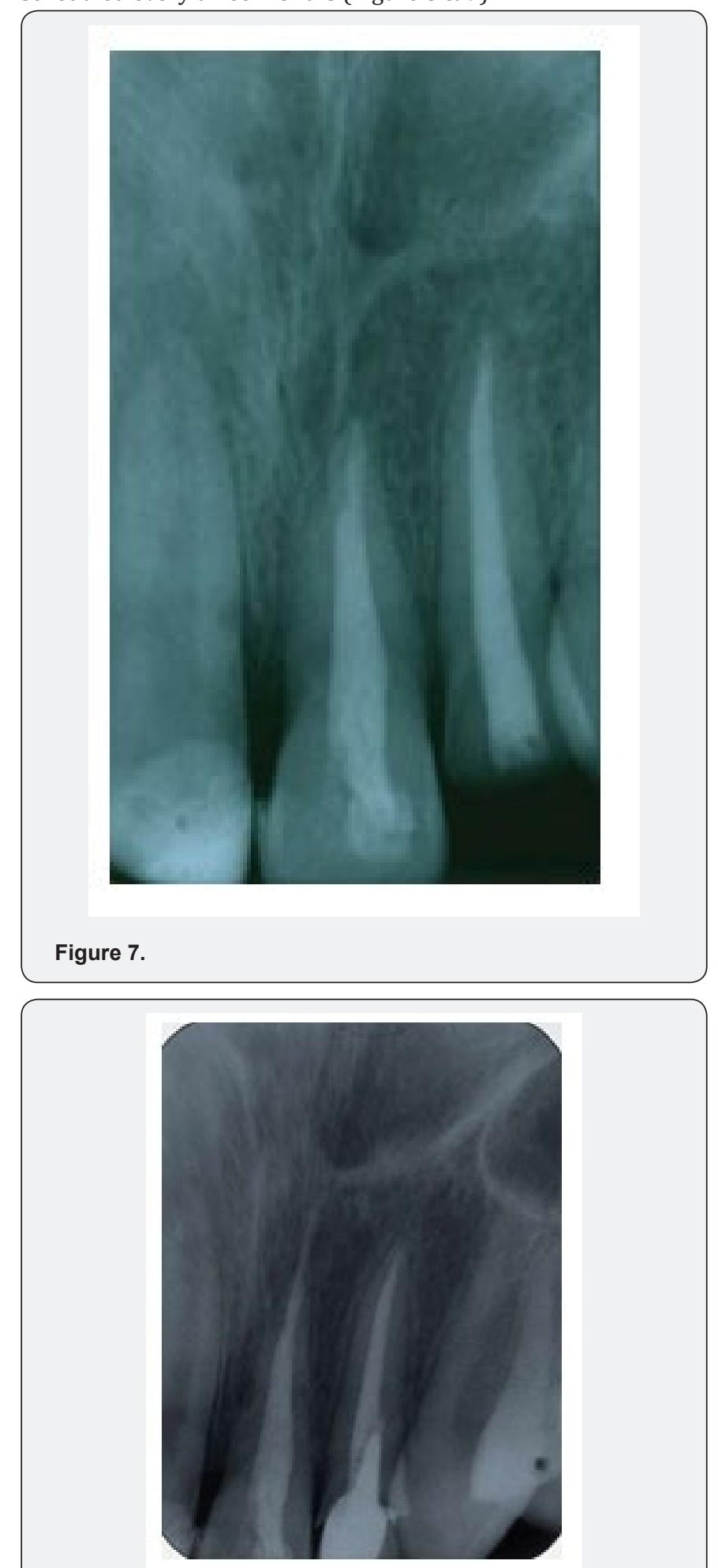

Figure 8. 


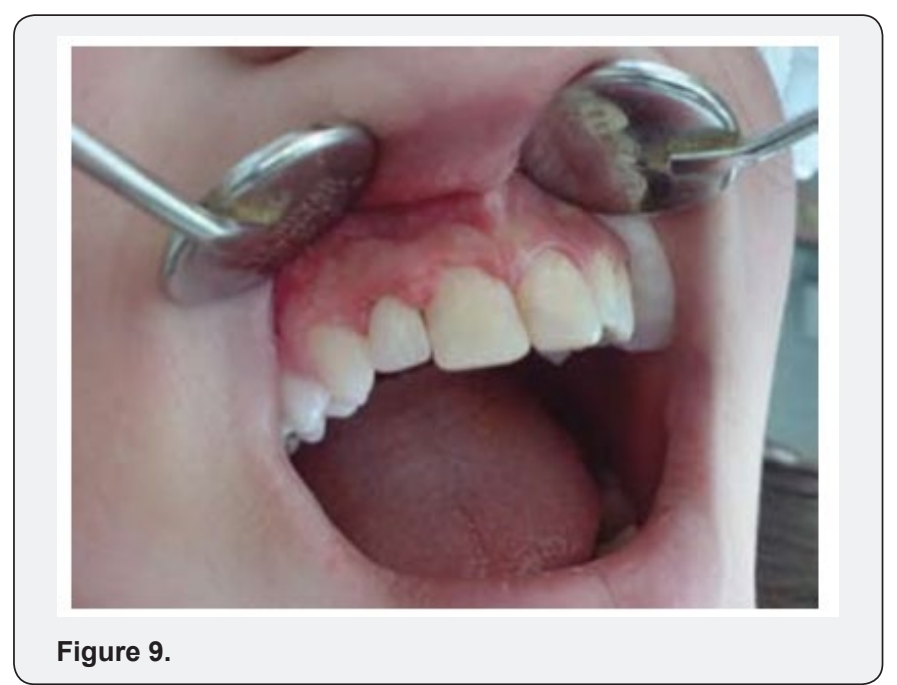

\section{Discussion}

Clinical practice shows that replantation success of avulsed teeth is certain in $4 \%-50 \%$ of cases. The main reasons for relatively great number of unsuccessful replantation are in the way the teeth are being treated until the replantation itself, as well as in the period of time prior the replantation. A contribution to this statement is the fact that success in replanting extracted and immediately replanted teeth in laboratory conditions is $100 \%$. Cvek et al. [4] emphasize that avulsed teeth being out of alveoli between 15 and 60 minutes, have considerably lower degree of post replanted repsorption if they were in physiological solution about 30 min before replantation [6]. During the replantation itself, it is of great importance that the cells of the tooth root suffer as little pressure as possible, so as not to be damaged against the alveoli wall. In that sense, it is inevitable to say that splint has relatively bad influence due to permanent pressure of one side of the tooth to alveoli.

During the treatment of the reported patient, we applied the rule that it is important to replant a tooth, regardless the time when it was brought, even if it was more than 2 hours after the injury, because, even then, there are chances, although minimal, to keep the tooth, and they are greater than in case of no replantation at all.

Literature data show that there is no need to rush with endodontic treatment of tooth root canal and filling with calcium hydroxide, because of increased incidence of apical ankylosis as a consequence of cytotoxic substances intrusion during canal treatment into Periodontal Ligament area (PDL). A compromised period of 7 to 10 days after an injury was established for endodontic treatment in order to prevent avulsed tooth pulp necrosis. All further delays extremely increase risk of post replantation necrosis and tooth loss.

In the reported case we decided to do treatment according to a specific situation - the fact that replantation was done more than 2 hours after the injury, which also increased the risk of fast developing necrosis processes, the fact that the patient was young with extremely voluminized pulp chamber, which also speeds up spreading of the inflammatory process to periodontal area (PDL). It is also worth mentioning that storing avulsed teeth in water or saliva should be avoided, because in these mediums PDL cells are damaged, increasing the root resorption. Milk is a suitable media for storing an avulsed tooth for only 15 to 20 minutes. It only prevents cell death in short term, but it does not have the ability to replenish them.

The period of wearing a splint is of extreme importance. The authors agree that due to complex reparative processes in replantation of completely disarticulated tooth, this period of time must not be less than 3 weeks and the maximum is 6 weeks, bearing the risk of possible post replantation complications, such as tooth ankylose or external resorption. The patient reported was wearing a splint for 4 weeks, what is an optimal interval for regeneration of periodontal tissue.

\section{Conclusion}

It is obligatory to perform tooth replantation, regardless the time of patient's arrival. This is also a way for a patient to gain confidence in the dentist who did their best for success of the treatment. There are 3 key moments during the complete treatment of an avulsed tooth important for the success of the therapy:

1. The time from avulsion to replantation: always do replantation, considering that possibilities of complications increase with longer period of time from avulsion to the therapy.

2. The moment of starting the endodontic treatment of the tooth root canal: the case reported in this study shows that positive results were obtained with pulp extirpation 24 hours after avulsion, and they are related to the period of one year after avulsion. The patient will be observed during the next three years.

3. Prolonged time of wearing a splint: positive results in the reported case show that optimal time for wearing a splint in tooth avulsion is 4 weeks.

\section{References}

1. Krasner P, Rankowh HJ (1995) New philosophy for the treatment of avulsed teeth. Oral Surg Oral Med Oral Pathol Oral Radiol Endod 79(5): 616-623.

2. Gonda F, Nagase M, Chen RB, Yabata H, Nakajima T (1990) Replantation: An analysis of 29 teeth. Oral Surg Oral Med Oral Pathol Oral Radiol Endod 70(5): 650-655.

3. Krasner P (1994) Modern treatment of avulsed teeth by emergency physicians. American Journal of Emergency Medicine 12(2): 241-246

4. Cvek M, Granath L, Hollender L (1974) Treatment of non-vital permanent incisors with calcium hydroxide. iii. Variations of occurrence of nakylosis of replanted teeth with duration of extra-alveolar period storage environment. Odontolrevy 25(1): 43-56. 
5. Kristerson l, Andreasen J (1983) The effect of splinting upon periodontal and pulpal healing after autotransplantation on mature and immature permanent incisors in monkeys. Int J Oral Surg 12(4): 239-249.

6. Andreasen J (1992) Atlas of replantation ant transplantation dog teeth. ( $1^{\text {st }}$ edn), Mediglobesa, Fribourg, Switzerland.

7. Trope M, Vesilsoy C, Koren L, Moshonov j, Friedman S (1992) Effects of different endodontic treatment protocols on periodontal repair and root resorption of replanted dog teeth. J Endod 18(10): 492-496.

8. Kinironsm J, Boyd DH, Greggta (1999) Inflammatory and replacement resorption in reimplanted permanent incisor teeth: a study of the characteristics of 84 teeth. Endod Dent Traumatol 15(6): 269-272.
9. Pohl Y, Filipi A, Kirschner H (2003) Extraoral and endodontic treatment by retrograde insertion on posts: a long-term study on replanted and transplanted teeth. Oral Surg Oral Med Oral Pathol Oral Radiol Endod 95(3): 355-363.

10. Blomlof L (1991) Milk and saliva as possible storage media for traumatically exarticulated teeth prior to replantation. Swed Dent J 8: 1-25

11. Trope M, Friedman S (1992) Periodontal healing of replanted dog teeth stored in Viaspan, milk, hanks balanced salt solution. Endod Dent Traumatol 8(5): 183-188. 